\title{
Toward the existence and uniqueness of solutions for fractional integro-differential equations under uncertainty
}

\begin{abstract}
The main contribution of the current paper is to obtain new results on the existence and uniqueness of the solution of fractional integro-differential equations under uncertainty with nonlocal conditions. For this purpose, we have used two basic tools, the contraction mapping principle and Krasnoselskii's fixed-point theorem. Indeed, we have considered the original problem involving fuzzy Caputo differentiability, together with fuzzy nonlinear condition.
\end{abstract}

Keyword: Fractional integro-differential equations; Uniqueness; Solution 